Claremont Colleges

Scholarship@Claremont

WM Keck Science Faculty Papers

W.M. Keck Science Department

9-1-2004

\title{
Human Stick Balancing: Tuning Lèvy Flights to Improve Balance Control
}

Juan Luis Cabrera

University of Chicago

John Milton

Claremont McKenna College; Pitzer College; Scripps College

\section{Recommended Citation}

Cabrera, Juan Luis, and John G. Milton. "Human Stick Balancing: Tuning Lèvy Flights to Improve Balance Control." Chaos 14.3 (2004): 691-698. DOI: 10.1063/1.1785453

This Article is brought to you for free and open access by the W.M. Keck Science Department at Scholarship @ Claremont. It has been accepted for inclusion in WM Keck Science Faculty Papers by an authorized administrator of Scholarship @ Claremont. For more information, please contact scholarship@cuc.claremont.edu. 


\title{
Human stick balancing: Tuning Lèvy flights to improve balance control
}

\author{
Juan Luis Cabrera \\ Laboratorio de Física Estadística, Centro de Física, IVIC, Aparado 21827, Caracas 1020A, Venezuela \\ John G. Milton \\ Department of Neurology and Committees on Neurobiology and Computational Neuroscience, MC 2030, \\ The University of Chicago, Chicago, Illinois 60637
}

(Received 24 May 2003; accepted 30 June 2004; published online 3 September 2004)

\begin{abstract}
State-dependent, or parametric, noise is an essential component of the neural control mechanism for stick balancing at the fingertip. High-speed motion analysis in three dimensions demonstrates that the controlling movements made by the fingertip during stick balancing can be described by a Lévy flight. The Lévy index, $\alpha$, is approximately 0.9 ; a value close to optimal for a random search. With increased skill, the index $\alpha$ does not change. However, the tails of the Lévy distribution become broader. These observations suggest a Lévy flight that is truncated by the properties of the nervous and musculoskeletal system; the truncation decreasing as skill level increases. Measurements of the cross-correlation between the position of the tip of the stick and the fingertip demonstrate that the role of closed-loop feedback changes with increased skill. Moreover, estimation of the neural latencies for stick balancing show that for a given stick length, the latency increases with skill level. It is suggested that the neural control for stick balancing involves a mechanism in which brief intervals of consciously generated, corrective movements alternate with longer intervals of prediction-free control. With learning the truncation of the Lévy flight becomes better optimized for balance control and hence the time between successive conscious corrections increases. These observations provide the first evidence that changes in a Lévy flight may have functional significance for the nervous system. This work has implications for the control of balancing problems ranging from falling in the elderly to the design of two-legged robots and earthquake proof buildings. (C) 2004 American Institute of Physics. [DOI: 10.1063/1.1785453]
\end{abstract}

The effects of state-dependent, i.e., parametric, noise are well recognized in the dynamics of physical ${ }^{1-6}$ and economic $^{7,8}$ systems. Less attention has been given to the fact that in the nervous system, the effects of noise are often state-dependent. For example, membrane noise reflects fluctuations in conductance and, hence, its effect on current (proportional to the product of conductance and driving potential) is state-dependent. ${ }^{9}$ Parametric noise underlies the spontaneous fluctuations in pupil size ${ }^{10-12}$ and plays important roles in motor ${ }^{13-16}$ and balance ${ }^{17-19}$ control. Stochastic forcing of an important control parameter across a stability boundary produces a type of bursting behavior known as on-off intermittency, ${ }^{20-22}$ i.e., periods of low amplitude fluctuations alternate with shorter intervals of higher amplitude fluctuations ("bursts"). Previously we demonstrated that on-off intermittency can be observed in stick balancing at the fingertip. ${ }^{17}$ Here we demonstrate that another nonlinear phenomenon also arises in stick balancing, namely the changes in speed made by the fingertip exhibit a special type of random walk, referred to as a Lévy flight, of the type known to be optimal for performing random searches. ${ }^{23-26}$ Although the hope has been that the detection of these phenomena will lead to the discovery of universal laws for the control of complex systems, as yet there has been no evidence to argue against the possibility that these statistical properties are merely epi-phenomena. ${ }^{27,28}$ Here we address this question by in- vestigating the changes in the statistical properties of the controlling movements as a subject becomes more skilled in the task of stick balancing. In this way we obtain the first evidence to suggest changes in a Lévy flight may have functional significance.

\section{INTRODUCTION}

The performance of a learned, highly skilled movement is traditionally regarded to be the outcome of a highly orchestrated activation pattern of motor and premotor neurons in which the properties of the peripheral musculoskeletal system play little role. A central concept is that of stability, i.e., the tendency to return to an original position after some perturbation has pushed the system away from it: postural control involves the stabilization of the upright position, gait the stabilization of periodic, or limit cycle, patterns of movement, and so on (e.g., Ref. 29).

Recent observations challenge this orderly picture of motor control. The regulation of movement and balance is not entirely under neural control. ${ }^{30}$ Intrinsic properties of the musculoskeletal system are essential for the stabilization of rapid ("ballistic") movements in both hexapodal invertebrates $^{31}$ and bipedal vertebrates. ${ }^{32,33}$ State-dependent noise, i.e., noise whose effects depend on the state of the system, is of fundamental importance for the excitation of motoneurons, ${ }^{14}$ goal-directed arm movements, ${ }^{15}$ bimanual coordination, ${ }^{16}$ and balance control. ${ }^{17-19}$ Finally, neural con- 
trol is not necessarily optimized for the stabilization of equilibria and limit cycles, but can be critically tuned near, or perhaps on, the boundaries that demarcate different dynamical behaviors. ${ }^{17,34-40,42}$

Important insights into motor control have been obtained from studies of stick balancing. ${ }^{17-19}$ This visuomotor task cannot be performed using memorized movement patterns, but requires continuous closed-loop control. ${ }^{43}$ The fluctuations in the controlled variable (vertical displacement angle) are intermittent, i.e., intervals of large amplitude alternate with intervals of smaller amplitude fluctuations. Parametric noise is introduced into this balancing task since the fingertip, i.e., the pivot point for the inverted pendulum, moves in a complicated manner. ${ }^{44,45}$ The probability that the time interval, $\delta t$, between successive corrective movements, i.e., those that decrease the vertical displacement angle, is equal to $\delta t, P(\delta t)$, exhibits a $-3 / 2$-power law, ${ }^{17}$ i.e.,

$$
P(\delta t) \propto \delta t^{-3 / 2} .
$$

The observation of a $-3 / 2$-power law implies that an essential feature of the control involves stochastic or chaotic forcing of a control parameter, such as the gain, across the stability boundary. ${ }^{17,20-22}$ It has been shown that this statedependent noise mechanism enables balance control to be maintained on time scales shorter than the neural latency for corrective movements. ${ }^{17}$

The concept of a random walk has provided important insights into the nature of the control of human balance, in particular postural control. ${ }^{46,47}$ Here we show that during stick balancing the movements of the fingertip exhibit a special kind of random walk referred to as a Lévy flight (for useful reviews, see Refs. 23-26, 48). A Lévy flight is a random walk in which the probability, $P(s)$, of a step of length, $s$, is $P(s) \sim s^{-\alpha}$. Here we demonstrate that the controlling movements for stick balancing at the fingertip are well described by a Lévy flight. With a few days practice, individuals can become much more skilled in the task of stick balancing at the fingertip. We take advantage of this effect of practice to study how the properties of the Lévy flight change when skill level changes. In this way we obtain the first evidence to suggest that Lévy flights can be tuned in order to optimize performance.

\section{EXPERIMENTAL METHODS}

Six subjects were healthy volunteers, ages $18-52$. These experiments were performed according to the principles of the Declaration of Helsinki and informed consent was obtained. The experimental protocol was approved by The University of Chicago Hospital institutional review board.

\section{A. Stick balancing}

Stick balancing was performed while the subject was seated comfortably in a chair. The subject was required to keep their back in contact with the chair at all times. When a person balances a stick at the fingertip under these conditions, the wrist and fingers are held rigid and the movements occur at the elbow and shoulder. ${ }^{17}$ Sticks were dowels having a diameter of $6.35 \mathrm{~mm}$ and a length of 39 and $62 \mathrm{~cm}$. The mass of the sticks was kept constant at $35 \mathrm{~g}$ by constructing dowels with different materials: $39 \mathrm{~cm}$ (aluminum, $2.504 \mathrm{~g} / \mathrm{cm}^{3}$ ), $62 \mathrm{~cm}$ (Garolite, Grade G-11, $1.751 \mathrm{~g} / \mathrm{cm}^{3}$ ). The use of light, short sticks accentuates the requirement for continuous visual feedback control by the nervous system and minimizes the role of proprioceptive inputs.

Reflective markers were attached to each end of the stick as described previously. ${ }^{17}$ The total mass of the reflective markers was $<0.5 \mathrm{~g}$. Two specialized motion-capture cameras detected infrared light reflected from these markers. The image projected onto the CCD of each camera determines two of the spatial coordinates: The third coordinate is determined by using triangulation techniques involving both cameras. The marker closest to the finger was used to measure the movements of the fingertip during stick balancing. Two different motion capture camera systems were used: ProReflex-240 and ProReflex-1000 (Qualisys, Inc.). The sampling frequency of each camera could be adjusted downwards from their maximum sampling frequencies of, respectively, 240 and $1000 \mathrm{~Hz}$. Spatial resolution was, respectively, $1 / 30000$ and 1/70 000 of field of view. Under our experimental conditions this translates to a spatial resolution of, respectively, 50 and $4 \mu \mathrm{m}$.

The characteristics of the power spectra of the fluctuations in the vertical displacement angle have been presented elsewhere. ${ }^{17,49}$ For all six subjects, the statistical properties for stick balancing indicated the presence of on-off intermittency. The power laws we describe here are not observed when the eyes are closed and the arm is moved to mimic stick balancing. ${ }^{17}$

The movements of the fingertip during stick balancing contain two contributions (Fig. 1): (1) Relatively slow translations of the pivot point for the inverted pendulum; and (2) small amplitude "corrective" movements. These kinematic features correspond, respectively, to the "rambling" and "trembling" movements of the center of pressure recorded during human postural sway. ${ }^{50,51}$

Our focus concerns the nature of the fast corrective movements. The time interval between successive corrective movements in both stick balancing ${ }^{17}$ and postural sway ${ }^{50}$ are typically much shorter than the latency of the involved neural reflexes. We interpreted these movements in terms of a random walk. The step size per unit time is equal to the speed, $V$. The change in speed, $\Delta V$, provides an estimate of how fast the hand can respond to changes in stick position and is equivalent to a high pass filtering of the time series. $^{38,52}$

Briefly, the change in position of the marker, $\Delta \vec{r}(t)$, in one time step, $\Delta t$, is

$$
\Delta \vec{r}(t)=\vec{r}(t+\Delta t)-\vec{r}(t),
$$

where the notation $\vec{r}$ denotes the position vector (inset in Fig. 1). All vectors were measured from a common reference point provided by the Qualisys measurement system. The magnitude of the mean speed, $V$, is

$$
V(t)=\left\|\frac{\Delta \vec{r}(t)}{\Delta t}\right\|
$$

where the notation $\|$.$\| denotes the norm. Hence$ 


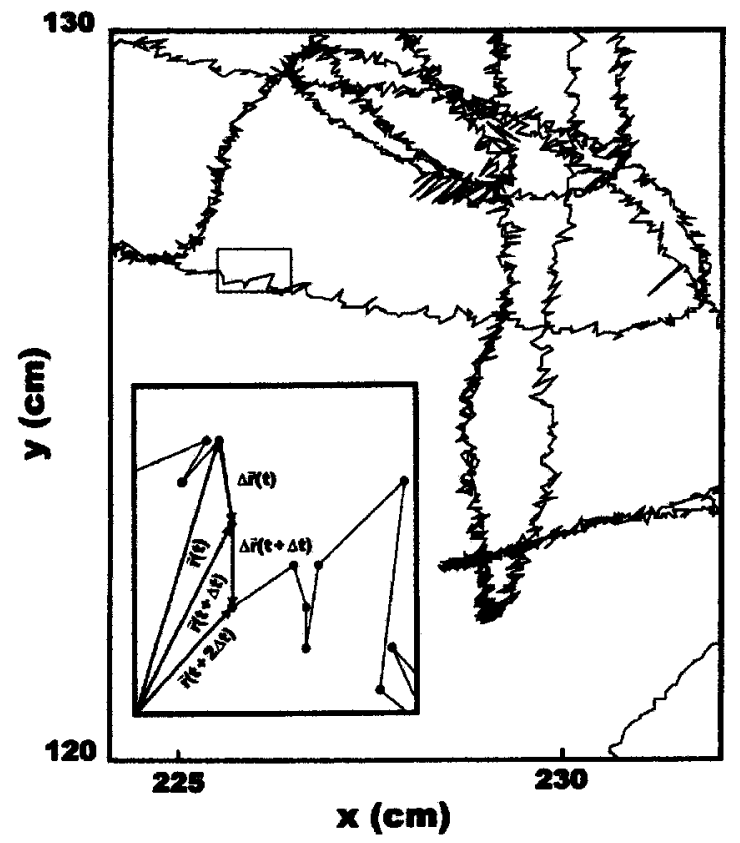

FIG. 1. Two-dimensional representation of the path traveled by the fingertip during stick balancing. The digitization timestep, $\Delta t$, was $1 \mathrm{~ms}$. Inset gives the definition of the position vectors, $\vec{r}$, used for the calculation of $\Delta V$ (see Experimental Methods). During $1 \mathrm{~ms}$ the values of $V$ range from 0.3 to 5 $\mathrm{mm} / \mathrm{s}$; the majority of the values were between 1 and $4 \mathrm{~mm} / \mathrm{s}$. Movements in the anterior-posterior direction are shown on the $y$ axis; side to side movements on the $x$ axis.

$$
\Delta V(t)=V(t+\Delta t)-V(t)
$$

where $V(t+\Delta t)=\|\vec{r}(t+\Delta t) / \Delta t\|$. It should be noted that $\Delta V$ is not equal to the acceleration. Using standard error propagation techniques we estimated that the error in the determination of $\Delta V$ calculated in this manner for data sampled at $1000 \mathrm{~Hz}$ was $\pm 2 \times 10^{-3} \mathrm{~m} / \mathrm{s}$.

\section{B. Lévy flights}

We characterized the changes in $\Delta V$ in terms of a Lévy flight (see also legends for Figs. 2 and 3). A Lévy flight is a random walk in which the length of each step per unit time, $\Delta t$, is taken from the power law distribution

$$
P(|\Delta V|>s, \Delta t) \approx s^{-\alpha},
$$

where $\alpha$ is the Lévy index. The probability density $P(\Delta V, \Delta t)$ converges after many steps to the Lévy stable distribution with index $\alpha$, namely

$$
L_{\alpha}(\Delta V, \Delta t)=\frac{1}{\pi} \int_{0}^{\infty} \exp \left(-\gamma \Delta t q^{\alpha}\right) \cos (q \Delta V) d q,
$$

where $\gamma$ is the scaling factor. ${ }^{53-57}$ A feature of this distribution is that for asymptotically large $\Delta V$ there is a power law decay of its density with exponent $\beta=\alpha+1$.

The exponent $\alpha$ was determined experimentally from the relationship

$$
P(0, \Delta t) \propto \Delta t^{-\alpha},
$$

where $P(0, \Delta t)$ is the probability of return, i.e., the probability when the change in speed is zero. ${ }^{56}$ We sampled the position of the lower marker on the balanced stick at $1000 \mathrm{~Hz}$
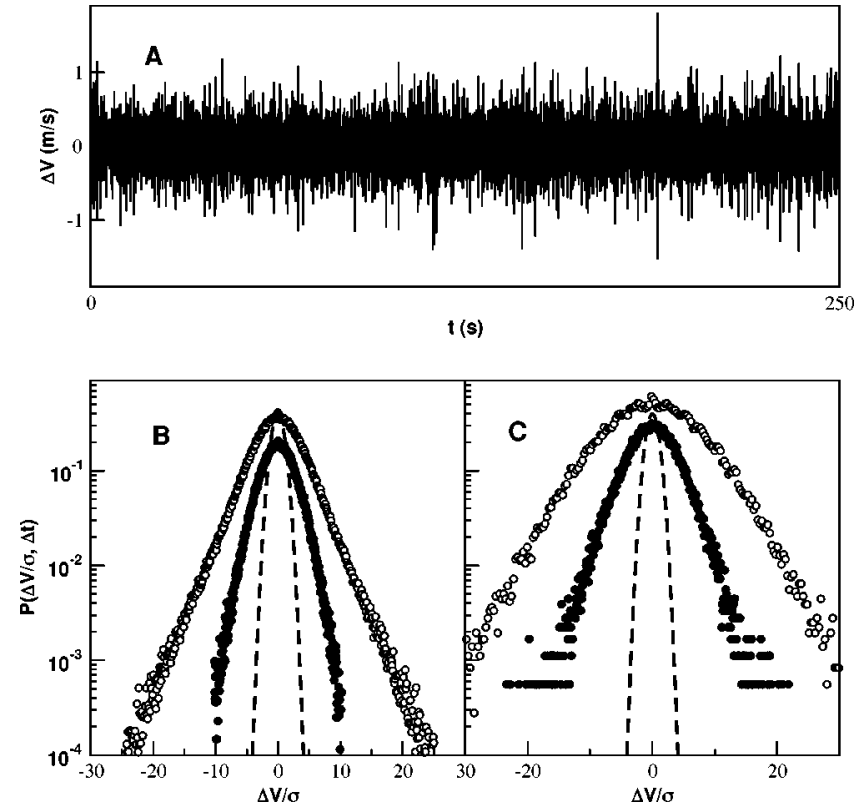

FIG. 2. Probability distributions of the change in speed of hand movements during stick balancing are not Gaussian-distributed. (a) Change in the speed of hand movements, $\Delta V$, as a function of time while a subject balances a stick at their fingertip (bottom reflective marker). The digitization step was 1 ms. (b) and (c) show the probability distribution, $P(\Delta V, \Delta t)$, for two less skilled (LS) subjects balancing a $62 \mathrm{~cm}$ stick. The changes in $\Delta V$ have been normalized to the standard deviation, $\sigma$, to facilitate comparison of the data to a Gaussian distribution (dashed line; calculated for the lower sampling frequency). The bin size was 6 . Data are shown for two sampling frequencies: $60 \mathrm{~Hz}(\Delta t \approx 16.7 \mathrm{~ms}, \bullet)$ and $120 \mathrm{~Hz}(\Delta t \approx 8.3 \mathrm{~ms}, \bigcirc)$. The data were pooled from repeated trials on the same day and the plots show mean distributions for the pooled trials.

$(\Delta t=0.001 \mathrm{~s})$ and then decimated this time series to obtain $P(0, \Delta t)$ as a function of $\Delta t$. Previous studies have suggested that $\approx 10^{5}-10^{6}$ data points are required to reliably distinguish Lévy distributions with $\alpha<1.2$ from a Gaussian distribution $(\alpha=2) .{ }^{54}$ In all cases we used $>5 \times 10^{5}$ data points to estimate $\alpha$. Since the data set is finite and is influenced by measurement noise, $P(0, \Delta t)$ depends on the bin size. The procedure described in Fig. 3 selects the optimum bin size that best filters out the effects of measurement noise.

\section{Skill acquisition}

The skill level was measured by determining the percentage of trials (typically $\geqslant 25$ ) that the stick remained balanced for at least $20 \mathrm{~s}$. All subjects practiced stick balancing for less than two hours before initial data were collected. Three subjects were excluded because they were able to balance only a few trials for the 39 and $62 \mathrm{~cm}$ sticks longer than $20 \mathrm{~s}$. We did not study these subjects further since the task of collecting sufficient data to test for Lévy phenomena would have been too tedious (see above). The data presented in this study are from the remaining three subjects [presented separately in Figs. 2(b), 2(c), and 3]. These subjects practiced stick balancing over longer periods to increase balancing skill. 

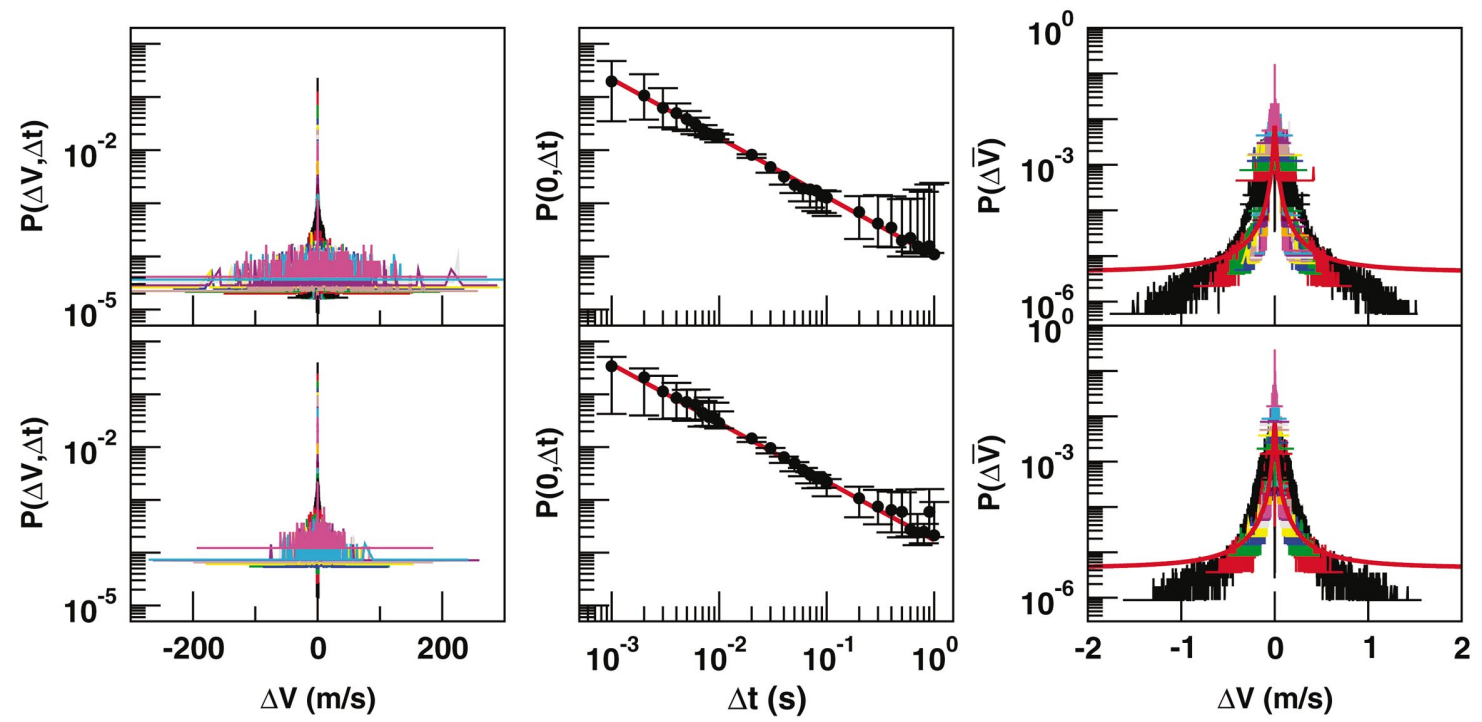

FIG. 3. (Color) Determination of $P(\Delta V, \Delta t)$ for two different stick lengths: (1) $39 \mathrm{~cm}$ stick (top row); (2) $62 \mathrm{~cm}$ stick (bottom row). Left hand column shows the distribution of $\Delta V$ for $\Delta t=0.001 \mathrm{~s}$ to $1 \mathrm{~s}$. The middle column shows a plot of $P(0, \Delta t)$ versus $\Delta t$. Since the data sets are finite the value of $P(0, \Delta t)$ depends on the bin size. The choice of bin size also affects the apparent noise level. The error bars for each $\Delta t$ show the maximum and minimum estimates of $P(0, \Delta t)$ as the bin size is changed from 0.0008 to $0.08 \mathrm{~m} / \mathrm{s}$. For all subjects we found that the bin size-related variation in $P(0, \Delta t)$ was smallest when $\Delta t$ was between 0.01 and $0.1 \mathrm{~s}$. Therefore, we used the values of $P(0, \Delta t)$ for this range of $\Delta t$ to estimate $\alpha$. The right hand column shows the rescaled $P(\Delta \bar{V})$ (see text for discussion). Each color in the left and right hand panels represents a different windowed data set.

\section{RESULTS}

Figure 2(a) shows the movements of the fingertip (lower stick reflector) during stick balancing. If the fingertip simply moved along a straight line at constant velocity, then $\Delta V$ $=0$. Clearly the movements of the fingertip are more complex (see also Fig. 1). Most of the changes in $\Delta V$ are quite small, i.e., less than $\pm 0.5 \mathrm{~m} / \mathrm{s}$ [Fig. 2(a)]. However, larger changes (some greater than $\pm 1 \mathrm{~m} / \mathrm{s}$ ) occur intermittently. The changes in the shape of the distribution of $\Delta V$, $P(\Delta V, \Delta t)$, when the sampling frequency is increased from 60 to $120 \mathrm{~Hz}$ [Figs. 2(b) and 2(c)] suggest that $\Delta V$ is not Gaussian distributed. ${ }^{5-57}$ When $\Delta V$ is normalized by the standard deviation, $\sigma, P(\Delta V, \Delta t)$ for a Gaussian-distributed variable (shown as dashed line in figure), the distribution falls abruptly and reaches zero at three times the standard deviation, i.e., at $\pm 3-4$ in Fig. 2.

\section{A. $\Delta V$ is Lévy distributed}

Stochastic processes involving parametric noise are frequently associated with power law distributions that have exponents typical for Lévy flights and Lévy distributions. ${ }^{3,7,8}$ Figure 3 shows that for two different stick lengths, $39 \mathrm{~cm}$ (top row) and $62 \mathrm{~cm}$ (bottom row) the fluctuations in $\Delta V$ measured for a subject skilled in stick balancing are described by a Lévy distribution. The slope of the log-log plot of $P(0, \Delta t)$ versus $\Delta t$ is linear for three decades and yields $\alpha \approx 0.9$ (we estimate a maximum uncertainty of \pm 0.05 ). The panels on the right hand side of Fig. 3 verify that for this value of the Lévy index, $P(\Delta V, \Delta t)$ rescales under the transformation $^{56}$

$$
P(\Delta \bar{V})=\frac{P(\Delta V, \Delta t)}{(\Delta t)^{-1 / \alpha}}
$$

where

$$
\Delta \bar{V}=\frac{\Delta V}{(\Delta t)^{1 / \alpha}} .
$$

The red line in Fig. 3 (right hand panels) is the theoretical Lévy distribution for $\alpha=0.9$ and scale factor $\gamma=0.02$. Although the fit of the Lévy distribution is good in the central part of the distribution, it obviously does not well describe the tails of the distribution. We interpreted this discrepancy as reflecting the effects of truncation. ${ }^{55-57}$ Truncation accounts for the fact that $\Delta V$ is limited by both the biomechanical properties of the musculoskeletal system and the effects of time-delayed feedback mechanisms. Thus we have

$$
P(\Delta V, \Delta t)=\left\{\begin{array}{l}
c_{1} L_{\alpha}(\Delta V, \Delta t) f(\Delta V) \text { if }|\Delta V|>\Pi \\
c_{2} L_{\alpha}(\Delta V, \Delta t) \quad \text { otherwise, }
\end{array}\right.
$$

where $c_{1}, c_{2}$ are normalization constants. The truncation function, $f(\Delta V)$, is typically a decreasing function of $\Delta V$ and the truncation threshold, $\Pi$, is the critical value of $\Delta V$ at which the distribution begins to deviate from $L_{\alpha}(\Delta V, \Delta t)$. Both $f(\Delta V)$ and $\Pi$ may also depend on $\Delta t$. This equation is consistent with current theories for the neural control of balance that emphasize a role for both open-loop and closedloop feedback mechanisms. ${ }^{46,58}$

\section{B. Skill acquisition}

Stick balancing skill steadily increases with practice. Figure 4 shows the effect of skill acquisition on $P(\Delta V, \Delta t)$ for a single individual. This individual practiced stick balancing for a total of $14 \mathrm{~h}$ over a 10-day period. Prior to this intensive period of practice this subject was able to balance $0 \%$ of trials using a $39 \mathrm{~cm}$ stick longer than $20 \mathrm{~s}$ and $45 \%$ of trials using a $62 \mathrm{~cm}$ stick [referred to herein as lower skill (LS)]; after practice, $33 \%$ and $100 \%$ of trials, respectively 


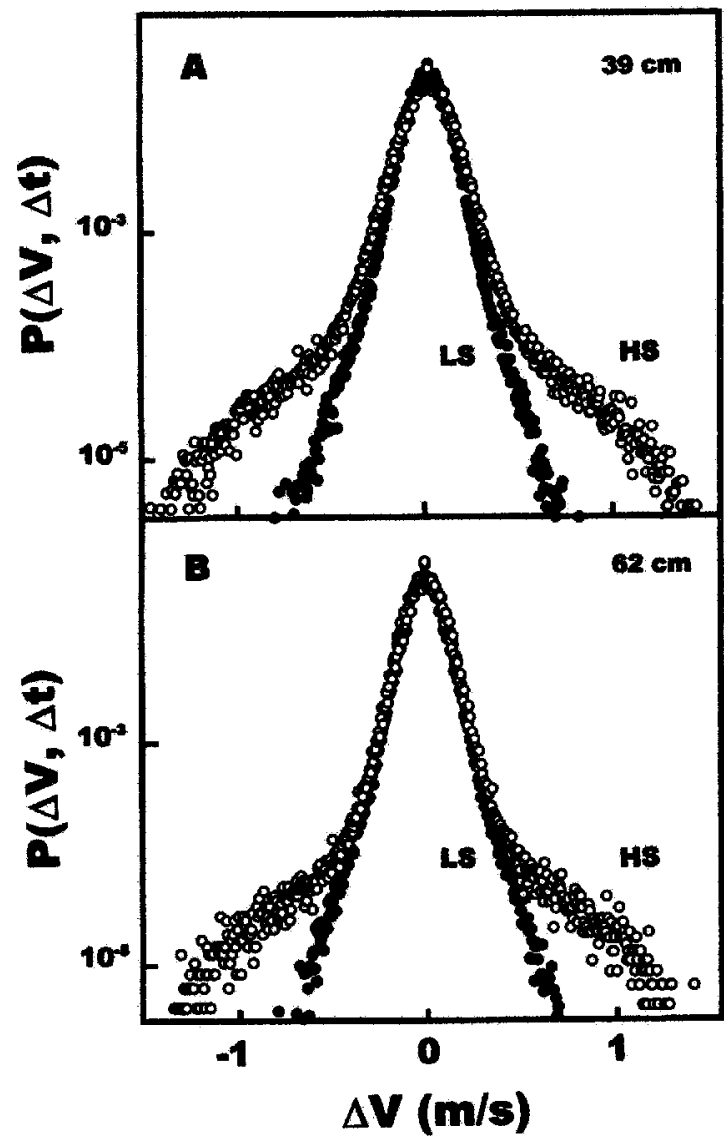

FIG. 4. Changes in the probability distribution, $P(\Delta V, \Delta t)$, as a function of expertise in stick balancing. The probability distributions were measured in the same subject at two different skill levels [LS (@) and HS (O)]: (a) $39 \mathrm{~cm}$ stick; (b) $62 \mathrm{~cm}$ stick. The sampling frequency was $1000 \mathrm{~Hz}(\Delta t=1 \mathrm{~ms})$ and the bin size was $6 \mathrm{~mm} / \mathrm{s}$. The data was pooled from repeated trials on the same day and the plots show mean distributions for the pooled trials. Total times of stick balancing were, respectively, for LS and HS, $39 \mathrm{~cm}(687$ s, $510 \mathrm{~s}), 62 \mathrm{~cm}(954 \mathrm{~s}, 767 \mathrm{~s})$.

[referred to herein as higher skill (HS)]. As can be seen $P(\Delta V, \Delta t)$ dramatically changes with skill level. In particular, $P(\Delta V, \Delta t)$ has a more pronounced tail for HS than LS. Thus increased skill is not simply because the nervous system is better able to anticipate the movements of the balanced stick: Better anticipation would be expected to decrease the need for higher $\Delta V$.

The values of $\alpha, \gamma$ did not change with the development of expertise in stick balancing. However, there was a slight change in $\Pi$ : for $39 \mathrm{~cm}$ stick $\Pi$ was 0.26 and 0.41 , respectively, for LS and HS; 0.26 and 0.40 for the $62 \mathrm{~cm}$ stick. These observations are in contrast to those observed in other physiological systems, e.g., the effects of disease on heart rate variability, in which the shape of the Lévy distribution remains constant and the power law changes as a result of changes in neural activity. ${ }^{59}$

The simplest model to explain the skill-related changes in $P(\Delta V, \Delta t)$ is that they are related to changes in truncation. The reason that $\alpha$ and $\gamma$ do not change is because these parameters were determined by the central part of $P(\Delta V, \Delta t)$ and this is not affected by the truncation.

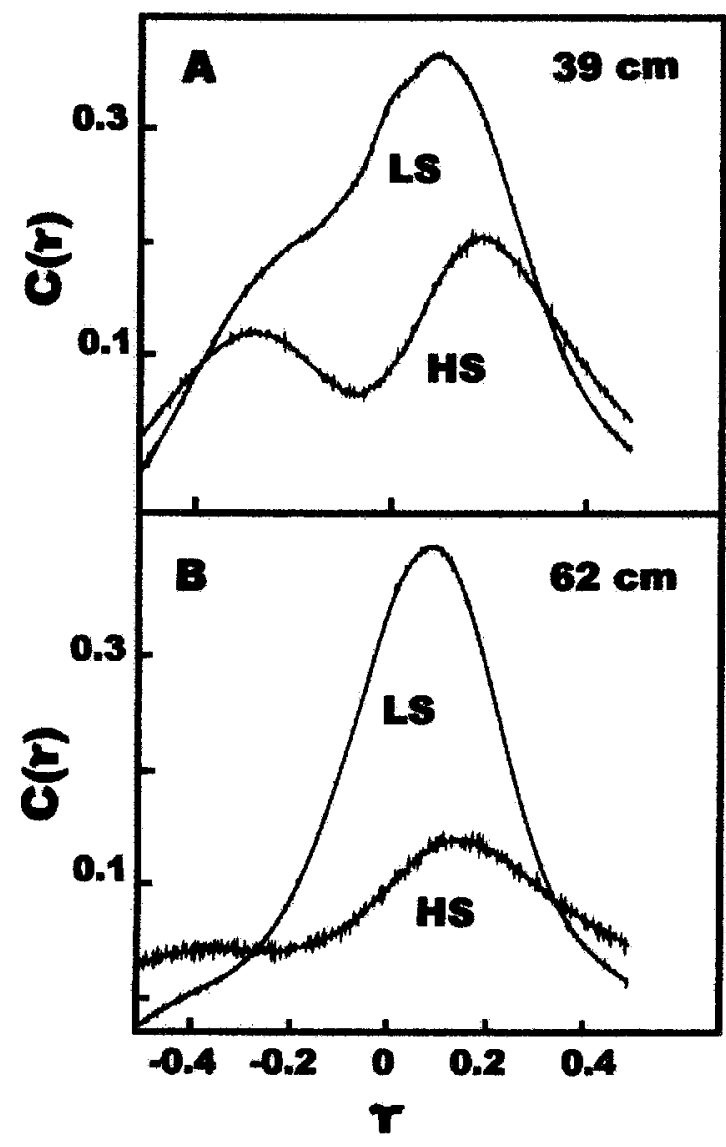

FIG. 5. Cross-correlations, $C(\tau)$, between the movements of the tip of the stick and the position of the fingertip change with skill level. (a) $39 \mathrm{~cm}$ stick; (b) $62 \mathrm{~cm}$ stick. The dominant peak in $C(\tau)$ indicates that for the $39 \mathrm{~cm}$ stick the response time increases from $\approx 110$ to $\approx 200 \mathrm{~ms}$ as skill level increases and for the $62 \mathrm{~cm}$ stick from $\approx 80$ to $\approx 150 \mathrm{~ms}$. Subject is the same as in Fig. 4.

\section{Truncating mechanisms}

It is well known that the importance of closed-loop feedback mechanisms decreases with increased motor skill. ${ }^{60}$ Let $f(\Delta V)$ be an exponentially decreasing function of $\Delta V$ with decay constant, $k$, i.e., $f(\Delta V) \approx \exp (-k|\Delta V|)$. Then the observed increase in the tails for $P(\Delta V, \Delta t)$ with skill level requires that $k$ decreases, i.e., the role of closed-loop feedback decreases ${ }^{57}$ In order to evaluate the possibility that the role of closed-loop feedback decreases with stick balancing skill, we computed the cross-correlation, $C(\tau)$, between the position of the tip of the balanced stick at time $t_{1}$ and the corrective movement made by the hand at time $t_{2}$, i.e., $\tau$ $=t_{2}-t_{1}$. The magnitude of $C(\tau)$ provides a measure of the importance of closed-loop feedback and the shift of the peak in $C(\tau)$ from $\tau=0$ gives an estimate of the response time for balance control.

Figure 5 shows $C(\tau)$ as a function of skill level. Since the dominant peak of $C(\tau)$ occurs for $\tau>0$, corrective movements in the fingertip occur in response to displacements in the tip of the stick. There are two differences in $C(\tau)$ between LS and HS: (1) The height of the dominant peak in $C(\tau)$ is smaller for the higher skill level; and (2) the displacement of the dominant peak of $C(\tau)$ from $\tau=0$ is greater for HS. The fact that $C(\tau)$ is different at the two skill 
levels supports our contention that the changes in $P(\Delta V, \Delta t)$ reflect changes in the role of closed-loop feedback in stick balancing.

The latencies of neural feedback control mechanisms for stick balancing can be estimated from the rightward shift in $C(\tau)$ from $C(0)$. In the untrained individual the dominant peak in $C(\tau)$ suggests that the time delay is of the order of 80-110 ms (see legend to Fig. 5). Although estimation of the time delay from measurements of $C(\tau)$ is known to be problematic it is of interest that these estimates are consistent with the critical delay, $\tau_{\text {cr }}$, determined for the stability of an inverted pendulum with time-delayed feedback. ${ }^{41}$ The critical delay gives the maximum value of the delay for which stable stick balancing is possible in the absence of noise. Measurements of the proficiency of stick balancing as a function of stick length indicate that there is an abrupt improvement in balancing skill once stick length exceeds $30 \mathrm{~cm}$ again suggesting that $\tau$ is of the order of $100 \mathrm{~ms}$. In motor control, time delays range from 50 to $100 \mathrm{~ms}$ for the regulation of muscle force ${ }^{61-63}$ and $77-230 \mathrm{~ms}$ for other manual tracking tasks in two dimensions. ${ }^{64}$

The rightward shift of the peak in $C(\tau)$ with increased skill suggests that the response time is increased at the higher skill level. A longer response time for HS was also found when the time delay was estimated using a delayed random walk algorithm. ${ }^{65}$ This increase in response time is surprising since for a given skill level a longer time delay is expected from Fitt's law ${ }^{66}$ to have a detrimental effect on the trade-off between movement speed and accuracy. ${ }^{67}$ However, a beneficial effect of delay on haptic spatial matching tasks has been reported. ${ }^{68}$ An alternative explanation is presented in the Discussion.

\section{DISCUSSION}

Previous studies have suggested that there is an element of predictive control in human stick balancing at the fingertip. ${ }^{43}$ However, the fact that survival statistics for stick balancing can be reproduced by a stochastic delay equation having only an unstable fixed point ${ }^{19}$ raises the possibility that the control is, at least in part, nonpredictive. This suggestion is supported by the observation that the controlling movements made by the fingertip can be described by a Lévy flight with $\alpha \sim 0.9$ and indicates that these movements have the characteristics of superdiffusion. ${ }^{26}$ Lévy flights with $\alpha$ $\approx 1$ (equivalently $\beta \approx 2$ ) have been shown to be optimal for random search patterns ${ }^{24-26}$ and have been detected in a variety of search tasks including animal foraging strategies ${ }^{23}$ and human eye movements during reading. ${ }^{69}$ This observation strongly suggests that the nervous system is not capable of predicting the movements of the balanced stick, but develops a foraging strategy.

An inverted pendulum can be stabilized by time-delayed feedback. ${ }^{17,41}$ However, we believe that the critical issue is not to identify the nature of the resultant attractor, but rather to understand why the stick eventually falls. ${ }^{19}$ Thus the critical questions are related to the first-passage times taken for a trajectory to escape from the basin of attraction. Lévy flights arise naturally in the context of the first passage time from an interval with absorbing boundaries. ${ }^{25}$

We have argued that the various power laws observed in stick balancing arise as effects of parametric noise. Indeed the combination of intermittency, Lévy flights, and Lévy distributions, has only been reported for certain types of stochastic dynamical systems with parametric noise. ${ }^{3}$ These effects of noise approximate the stochastic nature of the complex neural control systems involved in the regulation of movement and balance. Recently $-3 / 2$ power laws have been reported for the formation of avalanches ("forest fires") in feedforward-type neuronal networks. ${ }^{37,39}$ In some of these models, e.g., ${ }^{39}$ the probability of neural firing depends on the state of the neurons and hence can be considered to represent a state dependent perturbation. Thus it is possible that connections can be drawn between these phenomena and our observations on the effects of parametric noise on stick balancing.

Our observations imply that the Lévy flight (distribution) that describes the controlling movements made by the fingertip during stick balancing is truncated and that this truncation changes as expertise in this balancing task increases. Expertise in stick balancing improves as the nervous system is able to make bigger (faster) changes in speed, i.e., the distribution $P(\Delta V, \Delta t)$ develops longer tails. Closed-loop (sensory) feedback mechanisms provide one plausible mechanism whereby the truncation of the Lévy flight could be adjusted or tuned. This possibility, described by Eq. (4), is consistent with suggestions that skilled movement patterns emerge from an interaction between neural dynamics and musculoskeletal properties. ${ }^{30,70}$ Time delays have important effects on the properties of truncated Lévy processes even in the case that the autocorrelation function is zero. ${ }^{71}$

A tunable mechanism for balance control provides a simple explanation for the wide diversity in stick balancing skill between individuals (see METHODS). Variations in the effectiveness of closed-loop feedback mechanisms [reflected by, for example, $f(\Delta V, \Delta t)]$ and physical limitations imposed by differences in the musculoskeletal system [reflected by, for example, $\Pi(\Delta t)]$ limit the extent to which the Lévy distributed variable can be optimally tuned. This, in turn, leads to variations in the time that a stick can be, on average, balanced.

Our observation that the changes in speed for hand movements during stick balancing are described by a truncated Lévy distribution [Eq. (4)] is consistent with current theories for the neural control of balance that emphasize a role for both open-loop and closed-loop feedback mechanisms: ${ }^{46,58}$ Open-loop control predominates for small displacements with closed-loop control becoming operative once the displacement exceeds a certain threshold. Similarly in engineering contexts hybrid techniques are often employed to improve the survival of attractors having a finite basin of attraction: ${ }^{72}$ A safety net is built such that if a disturbance moves the system outside the basin of attraction, then it can be guided back by the application of a different control strategy.

Current neurophysiological theories for the development of expertise by the nervous system stress interplay between 
two processes: (1) An increasing reliance on the selfregulatory aspects of the motor task; and (2) a minimization of the role of mechanisms based upon intentionally directed corrective movements. ${ }^{73,74}$ The important point is that intentional control by the nervous system behaves as a resource limited quantity ${ }^{75,76}$ and consequently the nervous system has very limited abilities to control multiple tasks simultaneously. Consequently motor control strategies that emphasize prediction are likely to be less advantageous than nonpredictive control mechanisms since the latter frees up intentional resources to address other tasks. ${ }^{74}$ One possibility is that the nervous system may exert intentional control in an intermittent fashion, i.e., every so often the nervous system checks to see whether or not an intentional directed corrective movement is necessary. ${ }^{74,77}$ Evidence in favor of this conjecture is the observation that the latency for stick balancing increases with increased skill. With learning the prediction-free mechanisms lengthen the transients and hence the time between successive conscious checks lengthens. In this context the fact that stick balancing can be maintained for 500-600 ms even though vision has been blanked out is not necessarily evidence for predictive control, ${ }^{43}$ but may provide an estimate of the maximum time between successive intentional corrective movements. Recently it has been shown in a model of a delayed random walker with an unstable origin that increasing the delay increases the time for escape. ${ }^{78}$

Understanding the dynamic aspects of the development of expertise in a motor skill has important implications for both learning and rehabilitation. ${ }^{74}$ Although it has long been known that there is a power law relationship between expertise and the number of times a motor skill is repeated, ${ }^{73}$ the underlying neural mechanisms have yet to be identified even in the case of stick balancing at the fingertip. The recent development of virtual balancing tasks that involve the interaction between a human and a computer greatly facilitates the manipulation of key parameters ${ }^{18,43,79}$ together with noninvasive multimodal measurement methods, for example, electro-encephalography, electro-myography and functional magnetic resonance imaging, may enable these important aspects to be identified. Thus we anticipate that investigations of the paradigm of stick balancing will play an increasingly important role for uncovering the universal laws that enable to nervous system to develop expertise.

\section{ACKNOWLEDGMENTS}

We thank R. Knox and M. McClellan for technical assistance in performing these experiments and S. A. Campbell, D. Chialvo, C. W. Eurich, J. Guckenheimer, N. G. Hatsopoulos, L. P. Kadanoff, J. D. Hunter, R. McCrea, T. Ohira, S. Schaal, and G. Stépán for useful comments. This research was supported by grants from the Brain Research Foundation (BRF) and NIMH.

${ }^{1}$ J. L. Cabrera and F. J. de la Rubia, "Numerical analysis of transient behavior in the discrete random logistic equation with delay," Phys. Lett. A 197, 19-24 (1995).

${ }^{2}$ J. L. Cabrera and F. J. de la Rubia, "Analysis of the behavior of a non- linear delay discrete random logistic equation with delay," Int. J. Bifurcation Chaos Appl. Sci. Eng. 6, 1683-1690 (1996).

${ }^{3}$ O. S. Solomon and M. Levy, "Spontaneous scaling emergence in generic stochastic systems," Int. J. Mod. Phys. C 7, 745-751 (1996).

${ }^{4} \mathrm{~F}$. Rodelsperger, A. Censys, and H. Benner, "On-off intermittency in sin-wave instabilities," Phys. Rev. Lett. 75, 2594-2597 (1995).

${ }^{5}$ D. L. Feng, C. X. Yu, J. L. Xie, and W. X. Ding, "On-off intermittencies in gas discharge plasma," Phys. Rev. E 58, 3678-3685 (1998).

${ }^{6}$ T. John, R. Stannarius, and U. Behn, "On-off intermittency in stochastically driven electrohydrodynamic convection in nematics," Phys. Rev. Lett. 83, 749-752 (1999).

${ }^{7}$ A.-H. Sato, H. Takayasu, and Y. Sawada, "Power law fluctuation generator based on analog electrical circuit," Fractals 8, 219-225 (2000).

${ }^{8}$ H. Takayasu, A.-H. Sato, and M. Takayasu, "Stable infinite variance fluctuations in randomly amplified Langevin systems," Phys. Rev. Lett. 79, 966-969 (1997).

${ }^{9}$ F. S. Chance, L. F. Abbott, and A. D. Reyes, "Gain modulation from background synaptic input," Neuron 35, 773-782 (2002).

${ }^{10}$ L. Stark, F. W. Campbell, and J. Atwood, "Pupillary unrest: An example of noise in a biological servomechanism," Nature (London) 182, 857-858 (1958).

${ }^{11}$ J. G. Milton, A. Longtin, A. Beuter, M. C. Mackey, and L. Glass, "Complex dynamics and bifurcations in neurology," J. Theor. Biol. 138, 129147 (1989).

${ }^{12}$ A. Longtin, J. G. Milton, J. E. Bos, and M. C. Mackey, "Noise and critical behavior of the pupil light reflex at oscillation onset," Phys. Rev. A 41, 6992-7005 (1990).

${ }^{13} \mathrm{~K}$. Vasilakos and A. Beuter, "Effects of noise on a delayed visual feedback system," J. Theor. Biol. 165, 389-407 (1993).

${ }^{14}$ P. B. C. Matthews, "Relationship of firing intervals of human motor units to the trajectory of post-spike after-hyperpolarization and synaptic noise," J. Physiol. (London) 492.2, 597-628 (1996).

${ }^{15}$ C. M. Harris and D. M. Wolpert, "Signal-dependent noise determines motor planning," Nature (London) 394, 780-784 (1998).

${ }^{16}$ V. K. Jirsa, P. Fink, P. Foo, and J. A. S. Kelso, "Parametric stabilization of biological coordination: a theoretical model," J. Biol. Phys. 26, 85-112 (2000).

${ }^{17}$ J. L. Cabrera and J. G. Milton, "On-off intermittency in a human balancing task," Phys. Rev. Lett. 89, 158702 (2002).

${ }^{18}$ J. L. Cabrera, R. Bormann, C. Eurich, T. Ohira, and J. Milton, "Statedependent noise and human balance control," Fluct. Noise Lett. 4, L107L118 (2004).

${ }^{19}$ J. L. Cabrera and J. G. Milton, "Stick balancing: On-off intermittency and survival times," Nonlinear Science (to be published).

${ }^{20}$ A. S. Pikovsky, "On the interaction of strange attractors," Z. Phys. B: Condens. Matter 55, 145-154 (1984).

${ }^{21}$ N. Platt, E. A. Spiegel, and C. Tresser, "On-off intermittency: A mechanism for bursting," Phys. Rev. Lett. 70, 279-282 (1993).

${ }^{22}$ J. F. Heagy, N. Platt, and S. N. Hammel, "Characterization of on-off intermittency," Phys. Rev. E 49, 1140-1150 (1994).

${ }^{23}$ G. M. Viswanathan, V. Afanazyev, S. V. Buldyrev, E. J. Murphy, and H. E. Stanley, "Lévy flight search patterns of wandering albatrosses," Nature (London) 381, 413-415 (1996).

${ }^{24}$ G. M. Viswanathan, S. V. Buldyrev, S. Havlin, M. G. E. da Luz, E. P. Raposo, and H. E. Stanley, "Optimizing the success of random searches," Nature (London) 401, 911-914 (1999).

${ }^{25}$ S. V. Buldyrev, S. Havlin, A. Y. Kazakov, M. G. E. da Luz, E. P. Raposo, H. E. Stanley, and G. M. Viswanathan, "Average time spent by Lévy flights and walks on an interval with absorbing boundaries," Phys. Rev. E 64, 041108 (2001).

${ }^{26}$ D. Brockman and T. Giesel, "Lévy flights in inhomogeneous media," Phys. Rev. Lett. 90, 170601 (2003).

${ }^{27}$ C. Tsallis, S. V. F. Levy, A. M. C. Souza, and R. Maynard, "Statisticalmechanical foundation of the ubiquity of Lévy distributions in nature," Phys. Rev. Lett. 75, 3589-3593 (1995).

${ }^{28}$ H. E. Stanley, L. A. N. Amaral, J. S. Andrade, S. V. Buldyrev, S. Havlin, H. A. Makse, C-K. Peng, B. Suki, and G. Viswanathan, "Scale-invariant correlations in the biological and social sciences," Philos. Mag. B 77, 1373-1388 (1998).

${ }^{29}$ V. Zatsiorsky, Kinetics of Human Motion (Human Kinetics, Champaign, IL, 2002).

${ }^{30}$ H. J. Chiel and R. D. Beer, "The brain has a body: adaptive behavior emerges from interactions of nervous system, body and environment," Trends Neurosci. 20, 553-557 (1997). 
${ }^{31}$ D. L. Jindrich and R. J. Full, "Dynamic stabilization of rapid hexapod locomotion," J. Exp. Biol. 205, 2803-2823 (2002).

${ }^{32}$ A. J. Van Soest and M. F. Bobbert, "The contribution of muscle properties in the control of explosive movements," Biol. Cybern. 69, 195-204 (1993).

${ }^{33} \mathrm{R}$. Wagner and R. Blickhan, "Stabilizing function of skeletal muscles: an analytical investigation," J. Theor. Biol. 199, 163-179 (1999).

${ }^{34}$ J. A. S. Kelso, Dynamics Patterns: The Self-organization of Brain and Behavior (MIT Press, Boston, 1995).

${ }^{35}$ D. R. Chialvo and P. Bak, "Learning from mistakes," Neuroscience 90, 1137-1148 (1999).

${ }^{36} \mathrm{~J}$. Wakeling and P. Bak, "Intelligent systems in the context of surrounding environment," Phys. Rev. E 64, 051920 (2001).

${ }^{37}$ C. W. Eurich, J. M. Hermann, and U. A. Ernst, "Finite-size effects of avalanche dynamics," Phys. Rev. E 66, 066137 (2002).

${ }^{38}$ R. Segev, M. Benveniste, E. Hulata, N. Cohen, A. Palevski, E. Kapon, Y. Shapira, and E. Ben-Jacob, "Long term behavior of lithographically prepared in vitro neuronal networks," Phys. Rev. Lett. 88, 118102 (2002)

${ }^{39}$ J. M. Beggs and D. Plenz, "Neuronal avalanches in neocortical circuits," J. Neurosci. 23, 11167-11177 (2003).

${ }^{40}$ L. Moreau and E. Sontag, "Balancing at the border of instability," Phys. Rev. E 68, 020901 (2003).

${ }^{41}$ G. Stépán, Retarded Dynamical Systems: Stability and Characteristic Functions, Pitman Research Notes in Mathematics Series (Wiley, New York, 1989), Vol. 210, pp. 118-129.

${ }^{42}$ P. J. Treffner and J. A. S. Kelso, "Dynamic encounters: Long memory during functional stabilization," Ecological Psychol. 11, 103-137 (1999).

${ }^{43}$ B. Mehta and S. Schaal, "Forward models in visuomotor control," J. Neurophysiol. 88, 942-953 (2002).

${ }^{44}$ J. L. Bogdanoff and S. J. Citron, "Experiments with an inverted pendulum subject to random parametric excitation," J. Acoust. Soc. Am. 38, 447452 (1964)

${ }^{45}$ P. S. Landa, A. A. Zaikin, M. G. Rosenblum, and J. Kurths, "On-off intermittency phenomena in a pendulum with a randomly vibrating suspension axis," Chaos, Solitons Fractals 9, 157-169 (1998).

${ }^{46}$ J. J. Collins and C. J. De Luca, "Random walking during quiet standing," Phys. Rev. Lett. 73, 764-767 (1994).

${ }^{47}$ T. Ohira and J. G. Milton, "Delayed random walks," Phys. Rev. E 52, 3277-3280 (1995).

${ }^{48} \mathrm{M}$. Shlesinger, U. Frisch, and G. Zaslavsky, Lévy Flights and Related Phenomena in Physics (Springer-Verlag, New York, 1995).

${ }^{49}$ J. L. Cabrera and J. G. Milton, "Self-similarity in a human balancing task," Proceedings of the Second Joint EMBS/BMES Conference (Houston, TX), pp. 3-4 (2002).

${ }^{50} \mathrm{~V}$. M. Zatsiorsky and M. Duarte, "Instant equilibrium point and its migration in standing tasks: Rambling and trembling components of the stabilogram," Motor Control 3, 28-38 (1999).

${ }^{51}$ V. M. Zatsiorsky and M. Duarte, "Rambling and trembling in quite standing," Motor Control 4, 185-200 (2000).

${ }^{52}$ G. M. Jenkins and D. G. Watts, Spectral Analysis and its Applications (Holden-Hall, San Francisco, 1968), Chap. 7.

${ }^{53}$ D. Sornette, Critical Phenomena in Natural Sciences (Springer-Verlag, New York, 2000).

${ }^{54} \mathrm{R}$. Weron, "Levy-stable distributions revisited: Tail index $>2$ does not exclude the Levy-stable regime," Int. J. Mod. Phys. C 12, 209-223 (2001).

${ }^{55}$ I. Koponen, "Analytic approach to the problem of convergence of truncated Lévy flights towards the Gaussian stochastic process," Phys. Rev. E 52, 1197-1199 (1995)
${ }^{56}$ R. N. Mantegna and H. E. Stanley, "Scaling behavior in the dynamics of an economic index," Nature (London) 376, 46-49 (1995).

${ }^{57}$ H. M. Gupta and J. R. Campanha, "The gradually truncated Lévy flight: stochastic process for complex systems," Physica A 275, 531-543 (2000).

${ }^{58}$ C. W. Eurich and J. G. Milton, "Noise-induced transitions in human postural sway," Phys. Rev. E 54, 6681-6684 (1996).

${ }^{59}$ C.-K. Peng, J. Mietus, J. M. Hausdorff, S. Havlin, H. E. Stanley, and A. L. Goldberger, "Long-range anticorrelations and non-Gaussian behavior of the heartbeat," Phys. Rev. Lett. 70, 1343-1346 (1993).

${ }^{60} \mathrm{R}$. W. Pew, "Acquisition of hierarchial control over the temporal organization of a skill," J. Exp. Psychol. 71, 764-771 (1966).

${ }^{61}$ C. D. Marsden, P. A. Merton, and H. B. Morton, "Servo action in human voluntary movement," Nature (London) 238, 140-143 (1972).

${ }^{62}$ R. S. Johansson and G. Westling, "Roles of glabrous skin receptors and sensorimotor memory in automatic control of precision grip when lifting rougher or more slippery objects," Exp. Brain Res. 56, 550-564 (1984).

${ }^{63}$ E. Kunesh, F. Binkofski, and H.-J. Freund, "Invariant temporal characteristics of manipulative hand movements," Exp. Brain Res. 78, 539-546 (1989).

${ }^{64}$ K. C. Engel and J. F. Soechting, "Manual tracking in two dimensions," J. Neurophysiol. 83, 3483-3496 (2000).

${ }^{65}$ T. Ohira and R. Sawatari, "Delay estimation from noisy time series," Phys. Rev. E 55, 1-4 (1997).

${ }^{66} \mathrm{P}$. M. Fitts, "The information capacity of the human motor system in controlling the amplitude of movement," J. Exp. Psychol. 47, 381-391 (1954).

${ }^{67}$ I. S. MacKenzie and C. Ware, "Lag as a determinant of human performance in interactive systems," Proceedings of the ACM Conference on Human Factors in Computing Systems-INTERCHI'93 (ACM, New York, 1993), pp. 488-493.

${ }^{68}$ S. Zuidhoek, A. M. L. Kappers, R. H. J. van der Lubbe, and A. Postma, "Delay improves performance on a haptic spatial matching task," Exp. Brain Res. 149, 320-330 (2003).

${ }^{69}$ D. Brockman and T. Giesel, "The ecology of gaze shifts," Neurocomputing 32-33, 643-650 (2000).

${ }^{70}$ N. G. Hatsopoulos, "Coupling the neural and physical dynamics in rhythmic movements," Neurocomputing 8, 567-581 (1996).

${ }^{71}$ B. Podobnik, P. Ch. Ivanov, Y. Lee, A. Chessa, and H. E. Stanley, "Systems with correlations in the variance: Generating power law tails in probability distributions," Europhys. Lett. 50, 711-717 (2000).

${ }^{72} \mathrm{~J}$. Guckenheimer, "A robust hydrid stabilization strategy for equilibria," IEEE Trans. Autom. Control 46, 321-326 (1995).

${ }^{73}$ P. M. Fitts and M. I. Posner, Human Performance (Prentice Hall International, London, 1973).

${ }^{74}$ J. Milton, S. Small, and A. Solodkin, "On the road to automatic: Dynamic aspects of skill acquisition," J. Clin. Neurophysiol (to be published).

${ }^{75}$ M. A. Just, P. A. Carpenter, T. A. Keller, L. Emery, H. Zajac, and K. R. Thulborn, "Interdependence of nonoverlapping cortical systems in dual cognitive tasks," Neuroimage 14, 417-426 (2001).

${ }^{76}$ J. S. Rubinstein, D. E. Meyer, and J. E. Evans, "Executive control of cognitive processes in task switching," J. Exp. Psychol. Hum. Percept. Perform. 27, 763-797 (2001).

${ }^{77} \mathrm{M}$. A. Vince, "The intermittency of control movements and the psychological refractory period,” Br. J. Psychol. 38, 149-157 (1948).

${ }^{78}$ T. Hosaka, T. Ohira, J. G. Milton, and J. L. Cabrera, "Delayed random walk with a repulsive origin," Bull. Am. Phys. Soc. 49, 810 (2004).

${ }^{79}$ R. Bormann, J. L. Cabrera, J. G. Milton, and C. SW. Eurich, "Visuomotor tracking on a computer screen: An experimental paradigm to study the dynamics of motor control," Neurocomputing 58-60C, 517-523 (2004). 